Register. A copy of the October 17, 1997, Federal Register may be obtained, via mail by calling 202-512-1530 or accessed on-line through the US Government Printing Office at their web site: http://www.access.gpo.gov.

FROM: 1. Occupational Safety and Health Administration. Occupational exposures to tuberculosis. Federal Register October 17, 1997;201:54159-54308.

2. Occupational Safety and Health Administration. Instruction CPL2-106. February 9, 1996.

\section{Mycobacterium tuberculosis Transmission From Bronchoscope}

Two recent reports of Mycobacterium tuberculosis transmission from contaminated bronchoscopes underscore the importance of cleaning of lensed instruments, such as bronchoscopes, prior to disinfection or sterilization. Failure to clean, coupled with grossly inadequate disinfection practices, can result in infection transmission.

In one report, investigators from Johns Hopkins University School of Medicine identified two isolates with identical restriction fragment-length polymorphism (RFLP) patterns as part of an ongoing laboratory-based study of TB isolates.

The isolates were found to be from two patients diagnosed 6 months apart as having TB. Both isolates had a unique and identical 10-banded IS6110 RFLP pattern. Their only identifiable link was care at the same hospital.

An investigation was initiated, medical charts and bronchoscopic records were reviewed, and cleaning and disinfection of bronchoscopes was observed. It was determined that the first patient underwent bronchoscopy and was diagnosed as having TB. The second patient underwent bronchoscopy 2 days later and was diagnosed as having small cell carcinoma. Following 6 months of chemotherapy and radiation therapy, the second patient developed fever and an infiltrate of the right upper lobe of the lung. Bronchoscopic washings revealed acid-fast bacilli and grew $M$ tuberculosis. Both patients had undergone bronchoscopy with the same instrument in the same operating room with no intervening bronchoscopies. Bronchoscope cleaning and disinfection procedures were inconsistent with national guidelines. The authors concluded that a contaminated bronchoscope was the most likely source of $M$ tuberculosis transmission between these two patients.

In a second report, investigators from the CDC described an epidemiological investigation of nosocomial transmission of multidrug-resistant (MDR) TB. ${ }^{2}$ Eight patients with MDR TB were identified in South Carolina; all isolates were resistant to seven drugs and had matching DNA fingerprints (strain W1). Community links were identified for five patients (1-5). However, no links were identified for the other three patients (6-8) except being hospitalized at the same hospital as one community patient.

An outbreak investigation revealed that patient 5 (community-link patient) and patient 8, diagnosed April 1995 and November 1995, respectively, had clinical courses consistent with MDR TB, with smear-positive and culture-positive specimens and cavitary lesions on chest radiographs. Both died of MDR TB less than 1 month after diagnosis. Patients 6 and 7 (diagnosed in May 1995) each had one positive culture for MDR TB (specimens were collected during bronchoscopy). Patient 6 had a skin-test conversion after bronchoscopy. Neither patient 6 nor patient 7 had a clinical course consistent with MDR TB, neither was treated for MDR TB, and both are alive and well. No evidence of laboratory contamination of specimens, transmission on inpatient wards, or contact among patients was found. All four received bronchoscopies in May 1995; patients 6, 7, and 8 had bronchoscopies 1,12 , and 17 days, respectively, after patient 5. Observations revealed that bronchoscope cleaning was inadequate, and the bronchoscope was never immersed in disinfectant

The authors concluded that inadequate cleaning and disinfection of the bronchoscope after the procedure performed on patient 5 led to subsequent false-positive cultures in patients 6 and 7, transmission of infection to patient 6 , and active MDR TB in patient 8.

In both reports, direct observation of cleaning and disinfection of bronchoscopes revealed variation from institutional policy and national recommendations, including the "APIC Guideline for Infection Prevention and Control in Flexible Endoscopy" of the Association for Professionals in Infection Control and Epidemiology, Inc (APIC). ${ }^{3}$ As highlighted in the APIC guideline, disinfection cannot be achieved reliably without first accomplishing thorough mechanical cleaning. Even so, complex devices such as bronchoscopes remain a challenging device for reprocessing between patient use. Thorough cleaning of endoscopes can itself achieve a mean 4-log reduction in microbial load, and use of an appropriate germicide and good qualitycontrol monitoring can offer additional patient safety. ${ }^{4}$

FROM: 1. Michele TM, Cronin WA, Graham NM, Dwyer DM, Pope DS, Harrington S, et al. Transmission of Mycobacterium tuberculosis by a fiberoptic bronchoscope. Identification by DNA fingerprinting. JAMA 1997; 278(13):1093-1095.

2. Agerton T, Valway S, Gore B, Pozsik C, Plikaytis B, Woodley C, et al. Transmission of a highly drug-resistant strain (strain W1) of Mycobacterium tuberculosis: community outbreak and nosocomial transmission via a contaminated bronchoscope.JAMA1997;278(13):1073-1077.

3. Martin MA, Reicheldorfer M, APIC Guideline Committees. APIC guideline for infection prevention and control in flexible endoscopy. Am J Infect Control 1994;22:19-38.

4. Rutala WA, Weber D. FDA labeling requirements for disinfection of endoscopes: a counterpoint. Infect Control Hosp Epidemiol 1995;16:231-235.

\section{MRSA in A ustralia}

Despite vigorous attempts at eradication over the last 20 years, methicillin-resistant Staphylococcus aureus (MRSA) continues to be a major nosocomial pathogen in Australian acute-care institutions, reports Dr. McDonald from the Geelong Hospital in Victoria, Australia. The epidemiology of hospital spread is now well-characterized; infected and colonized patients provide the primary reservoirs, and transmis- 
sion mainly is via hospital staff. MRSA remains endemic in most of Australia's large urban teaching hospitals; occasional outbreaks also occur, especially in intensive-care areas. The level of MRSA infection often is indicative of the total rate of nosocomial infection within an institution and may reflect overcrowding, heavy workloads, and understaffing of wards.

Standard precautions, isolation and cohorting of infected and colonized patients, screening of staff, handwashing campaigns, nasal eradication policies, and increased staff education all have been tried, with variable success. There is no universal formula, and local problems require local solutions plus commitment of local resources.

Dr. McDonald suggests that preventing surgical infection with MRSA first requires the application of surgical principles and then compliance with the national recommendation against routine prophylaxis with vancomycin.

FROM: McDonald M. The epidemiology of methicillinresistant Staphylococcus aureus: surgical relevance 20 years on. Aust N Z J Surg 1997;67:682-685.

\section{Laboratory Identification of VRE}

Investigators from the CDC recently reported the findings of a study to determine whether hospital-based clinical laboratories conducting active surveillance for vancomycinresistant enterococci (VRE) in three San Francisco Bay-area counties (San Francisco, Alameda, and Contra Costa counties) were reporting vancomycin resistance accurately.

Five vancomycin-resistant enterococcal strains and one vancomycin-susceptible ß-lactamase-producing Enterococcus were sent to $31(97 \%)$ of 32 laboratories conducting surveillance. Each strain was tested by the laboratory's routine antimicrobial susceptibility testing method. An Enterococcus faecium strain with high-level resistance to vancomycin (minimum inhibitory concentration [MIC], $512 \mu \mathrm{g} / \mathrm{mL}$ ) was reported correctly as resistant by $100 \%$ of laboratories; an $E$ faecium strain with moderate-level resistance (MIC, 64 $\mu \mathrm{g} / \mathrm{mL}$ ) was reported correctly as resistant by $91 \%$ of laboratories; two strains of Enterococcus faecalis with low-level resistance (MIC, $32 \mu \mathrm{g} / \mathrm{mL}$ ) were reported correctly as resistant by $97 \%$ and $56 \%$ of laboratories, respectively. An Enterococcus gallinarum strain with intrinsic low-level resistance (MIC, $8 \mu \mathrm{g} / \mathrm{mL}$ ) was reported correctly as intermediate by $50 \%$ of laboratories. A B-lactamase-producing $E$ faecalis isolate was identified correctly as susceptible to vancomycin by $100 \%$ of laboratories and as resistant to penicillin and ampicillin by $68 \%$ and $44 \%$ of laboratories, respectively; all $23(74 \%)$ laboratories that tested for ß-lactamase recognized that it was a $ß$-lactamase producer.

This survey indicates that, for clinically significant enterococcal isolates, laboratories in the San Francisco Bay area accurately identify high-level vancomycin resistance; however, there are problems in detecting low-to-moderate level vancomycin resistance. The authors suggest that increasing accuracy of detection and prompt reporting of these isolates and investigation of cases are the next steps in the baffle for control of the spread of vancomycin resistance.

FROM: Rosenberg J, Tenover FC, Wong J, Jarvis W, Vugia DJ. Are clinical laboratories in California accurately reporting vancomycin-resistant enterococci? / Clin Microbial 1997;35:2526-2530.

\section{Conference on Emerging Infectious Diseases}

The Centers for Disease Control and Prevention, the Council of State and Territorial Epidemiologists, the American Society for Microbiology, and the CDC Foundation, together with more than 50 other co-sponsors, will present the International Conference on Emerging Infectious Diseases on March 8-11, 1998, in Atlanta, Georgia. The purpose of the conference is to encourage the exchange of scientific and public health information on global emerging infectious disease issues, increase awareness, identify program gaps, and enhance partnerships in addressing emerging infectious diseases.

The meeting will consist of plenary sessions, symposia, roundtables with invited speakers, presentations on emerging infection activities, oral and poster presentations based on submission of an accepted abstract, and exhibits. Major topics will include current work on surveillance, epidemiology, research, communications, training, and prevention and control of emerging infectious diseases, as well as topics related to emergency preparedness and response.

Abstracts should address new, re-emerging, or drugresistant infectious diseases that affect human health, and such topics as foodborne diseases, antimicrobial resistance, infectious diseases transmitted by animals and arthropods, infections acquired in healthcare settings, infectious diseases in immunodeficient persons, infectious diseases in hard-to-reach and other at-risk populations, infectious causes of chronic diseases, blood safety, host genetics, vaccines, global climate change, and immigration and travel.

Deadline for submission of abstracts is October 31 , 1997. Register early as attendance will be limited to 2,500 participants. Additional information on abstract submission and registration can be obtained at www.asmusa.org, by sending an e-mail message to meetinginfo@asmusa.org, or by calling 202-942-9248. Proceedings of the conference will be published in the Emerging Infectious Diseases journal.

Additional news items in this issue: TB Skin-Test Conversion Rates Among Exposed Hospital Workers, page 824; Pyrogenic Reactions Following Cardiac Catheterization, page 871 . 